\title{
Evaluation of the Effect of Sigmoid-Shaped Interventricular Septum on Left Ventricular Systolic Function in Patients with Essential Hypertension by Two-Dimensional Speckle Tracking Echocardiography
}

\author{
Zhifan Zhang1, Wei Xu'², Yudong Peng², Hong Zhang2, Qi Zhang1 \\ ${ }^{1}$ Department of Medicine, Yangtze University, Jingzhou, China \\ ${ }^{2}$ Department of Ultrasound, The First Affiliated Hospital of Yangtze University, Jingzhou, China \\ Email:*673059878@qq.com
}

How to cite this paper: Zhang, Z.F., Xu, W., Peng, Y.D., Zhang, H. and Zhang, Q. (2020) Evaluation of the Effect of Sigmoid-Shaped Interventricular Septum on Left Ventricular Systolic Function in Patients with Essential Hypertension by Two-Dimensional Speckle Tracking Echocardiography. Yangtze Medicine, 4, 62-69. https://doi.org/10.4236/ym.2020.41006

Received: November 2, 2019

Accepted: March 27, 2020

Published: March 30, 2020

Copyright $\odot 2020$ by author(s) and Scientific Research Publishing Inc. This work is licensed under the Creative Commons Attribution International License (CC BY 4.0).

http://creativecommons.org/licenses/by/4.0/

\begin{abstract}
Objective: To evaluate left ventricular regional and global systolic function by measuring left ventricular longitudinal strain (LS) in hypertensive patients with sigmoid-shaped interventricular septum (SIS) by two-dimensional speckle tracking (2D-STE); in order to explore whether the sigmoid-shaped interventricular septum affects the left ventricular systolic function in patients with hypertension. Methods: Routine echocardiographic parameters were measured in 30 hypertensive patients with SIS (SIS group) and 30 hypertensive patients without SIS (non-SIS group). The left ventricular segments and global LS were measured by 2D-STE, and the two sets of parameters were compared. Results: The value of the thickness of the basal segment of the interventricular septum (IVSBT), the thickness of the middle segment of the interventricular septum (IVSMT) and the ratio of the basal segment of the ventricular septum to the middle segment of the interventricular septum (IVSBT/IVSMT) in SIS group was higher than that in non-SIS group. However, the value of left ventricular outflow tract diameter (LVOTD) in SIS group was lower than that in non-SIS group. There was a significant difference between the two groups (all $\mathrm{P}<0$ 05). The LS values of the basal segment of the left ventricular anterior wall and the posterior wall of the left ventricle in the SIS group were lower than those in the non-SIS group. The difference was statistically significant (all $\mathrm{P}<0.05)$. Conclusion: SIS affects left ventricular regional systolic function of patients with hypertension. 2D-STE can early evaluate left ventricular longitudinal systolic function in hypertensive patients with SIS.
\end{abstract}




\section{Keywords}

Sigmoid-Shaped Interventricular Septum, Hypertension, Left Ventricular Systolic Function, Two-Dimensional Speckle Tracking Echocardiography, Echocardiography

\section{Introduction}

Sigmoid-shaped interventricular septum (SIS) was first proposed by Goor et al. [1] in 1969. It refers to the local thickening and protruding to the left ventricular outflow tract at the base of the interventricular septum, resulting in the shape of S-shaped blood flow through the left ventricular outflow tract. The Framingham Heart Research Center showed that the total prevalence rate of SIS was $1.5 \%$, compared with $17.8 \%$ of subjects aged 85 and older [2]. There is a close relationship between SIS and hypertension. In the observational study of SIS, the prevalence rate of hypertension is often between 55\% and 85\% [2] [3] [4]. So far, there is no consensus on the clinical significance of SIS [5]. It is not clear whether SIS affects left ventricular systolic function in patients with hypertension. As a non-invasive technique for quantitative evaluation of myocardial motion, two-dimensional speckle tracking echocardiography (2D-STE) has no angle dependence and can accurately evaluate left ventricular myocardial motion function [6]. The purpose of this study was to evaluate the left ventricular regional and global systolic function in hypertensive patients with SIS by 2D-STE, and to explore whether SIS affects the left ventricular systolic function in hypertensive patients.

\section{Data and Methods}

\subsection{Subjects}

Thirty patients with essential hypertension with SIS (SIS group) examined in the Department of Ultrasonography, First Affiliated Hospital of Yangtze University from March to August 2019 were selected, including 17 males and 13 females, aged $(64.43 \pm 7.48)$ years. At the same time, 30 patients with essential hypertension without SIS (non-SIS group) matched with SIS group were selected as control group, including 16 males and 14 females, aged $(60.67 \pm 8.59)$ years. Inclusion criteria: with reference to the Chinese guidelines for the Prevention and treatment of Hypertension (revised in 2018) [7]: That is, without antihypertensive drugs, systolic blood pressure $\geq 140 \mathrm{mmHg}$ and/or diastolic blood pressure $\geq 90 \mathrm{mmHg}$. Previous history of hypertension, taking antihypertensive drugs, blood pressure < 140/90 $\mathrm{mmHg}$, can also be diagnosed with hypertension. The SIS group also met the diagnostic criteria of SIS, according to the criteria of Framingham Cardiac Research Center: 1) Visual evaluation of basal segment hypertrophy of interventricular septum; 2) The thickness of basal segment of interventricular septum $\geq 1.4 \mathrm{~cm} ; 3$ ) The ratio of the basal segment of the ventri- 

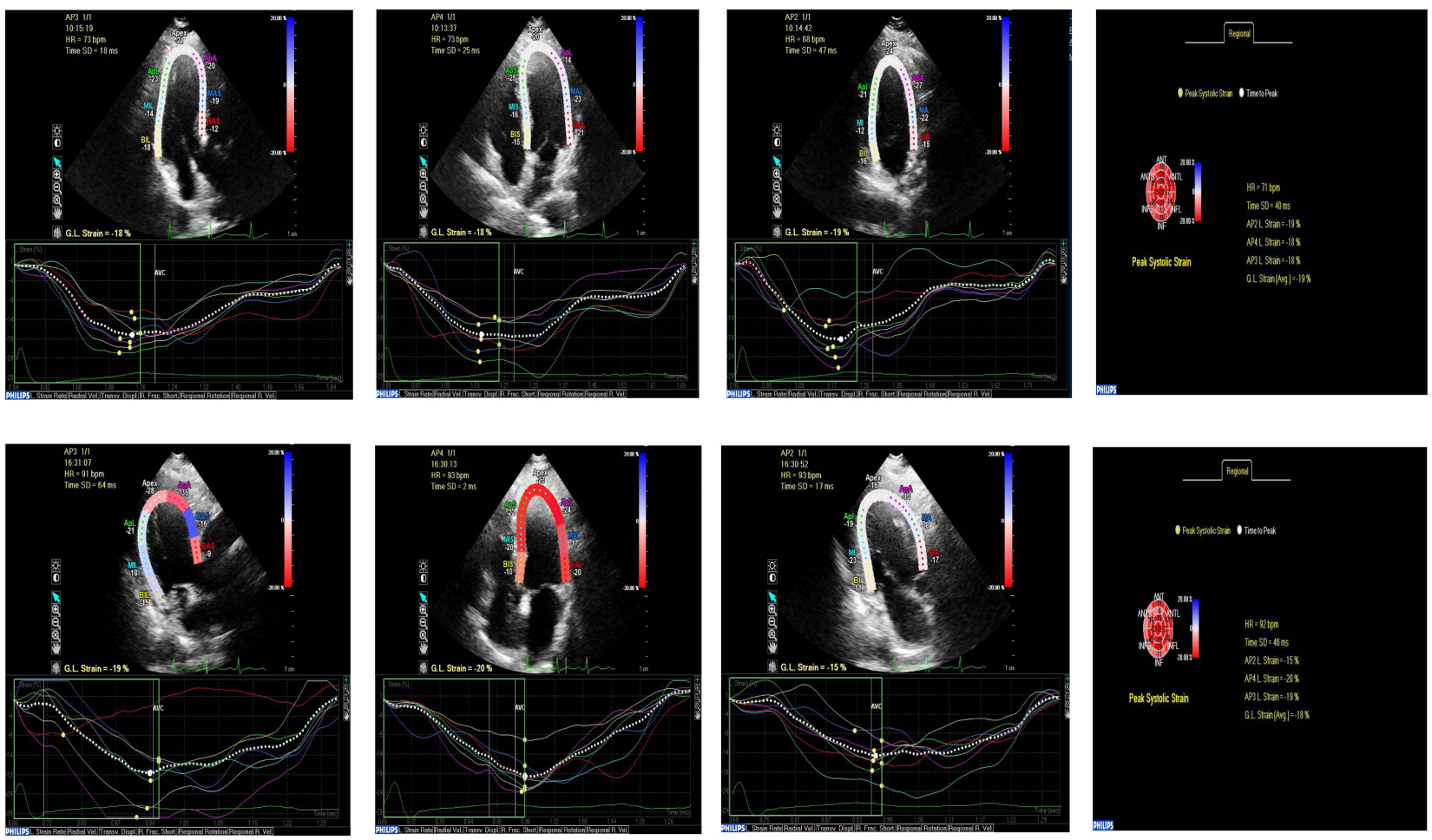

Figure 1. Global and segmental left ventricular LS curves and bovine eye diagrams on apical three-chamber, four-chamber and two-chamber sections in non-SIS group and SIS group. (A): non-SIS group; (B): SIS group. 
fraction (LVEF) by biplane Simpson. Adjust the frame rate of the instrument $>$ 60 frames/s, collect the two-dimensional dynamic images of the standard left ventricular apical four-chamber, three-chamber and two-chamber sections for three consecutive cardiac cycles, store them and export them to the Q-LAB workstation for 2D-STE offline analysis. The longitudinal strain (LS) of the whole and each segment on the three-chamber, four-chamber and two-chamber section strain curves of the left ventricular apex were measured. After the analysis of the three sections, the software will automatically generate a bovine eye diagram showing each segment.

\subsection{Statistical Analysis}

The data were analyzed and processed by SPSS 19.0 statistical software package, and the measurement data were expressed as mean \pm standard deviation $(\bar{x} \pm$ s). Independent sample t-test was used to compare with the two groups. The difference was statistically significant $(\mathrm{P}<0.05)$.

\section{Results}

\subsection{Comparison of Clinical Data and Echocardiographic Parameters between Non-SIS Group and SIS Group}

There was no significant difference in LVEF, LVPWT, LVOTV, Age, HR, SBP and DBP between the two groups ( $P>0.05)$. The value of IVSBT, IVSMT and IVSBT/IVSMT in SIS group was higher than that in non-SIS group, while the value of LVOTD in SIS group was lower than that in non-SIS group. There was significant difference between the two groups $(\mathrm{P}<0.05)$. See Table 1.

\subsection{Comparison of Ultrasonic Two-Dimensional Spot Tracking Parameters between Non-SIS Group and SIS Group}

As shown in Table 2, compared with the non-SIS group, the LS of the basal

Table 1. Comparison of general data between non-SIS groups and SIS groups $(\bar{X} \pm s)$.

\begin{tabular}{|c|c|c|c|c|c|c|c|c|}
\hline Groups & Age (years) & \multicolumn{2}{|c|}{ HR (beats/min) } & \multicolumn{2}{|c|}{ SBP $(\mathrm{mmHg})$} & \multicolumn{2}{|c|}{$\mathrm{DBP}(\mathrm{mmHg})$} & LVEF (\%) \\
\hline non-SIS groups $(\mathrm{n}=30)$ & $60.67 \pm 8.59$ & \multicolumn{2}{|c|}{$77.0 \pm 8.59$} & \multicolumn{2}{|c|}{$141.89 \pm 12.92$} & \multicolumn{2}{|c|}{$84.78 \pm 7.08$} & $59.0 \pm 1.41$ \\
\hline SIS groups $(\mathrm{n}=30)$ & $64.43 \pm 7.48$ & \multicolumn{2}{|c|}{$77.86 \pm 11.61$} & \multicolumn{2}{|c|}{$143.21 \pm 11.28$} & \multicolumn{2}{|c|}{$86.14 \pm 13.30$} & $59.14 \pm 2.59$ \\
\hline$P$ value & 0.279 & \multicolumn{2}{|l|}{0.851} & \multicolumn{2}{|c|}{0.797} & \multicolumn{2}{|c|}{0.781} & 0.882 \\
\hline Groups & IVSBT (mm) & IVSMT (mm) & \multicolumn{2}{|c|}{ IVSBT/IVSMT } & \multicolumn{2}{|c|}{ LVPWT (mm) } & LVOTD (mm) & $\operatorname{LVOTV}(\mathrm{cm} / \mathrm{s})$ \\
\hline non-SIS groups $(\mathrm{n}=30)$ & $12.22 \pm 0.67$ & $9.33 \pm 0.87$ & \multicolumn{2}{|c|}{$1.32 \pm 0.13$} & \multicolumn{2}{|c|}{$9.33 \pm 0.71$} & $20.64 \pm 2.47$ & $82.22 \pm 17.71$ \\
\hline SIS groups $(n=30)$ & $15.50 \pm 1.09$ & $10.29 \pm 0.99$ & \multicolumn{2}{|c|}{$1.517 \pm 0.15$} & \multicolumn{2}{|c|}{$10.21 \pm 1.37$} & $17.75 \pm 1.91$ & $94.91 \pm 20.66$ \\
\hline$P$ value & 0.000 & 0.028 & \multicolumn{2}{|c|}{0.005} & \multicolumn{2}{|c|}{0.09} & 0.017 & 0.228 \\
\hline
\end{tabular}

Note: Date are expressed as mean \pm SD. SIS, sigmoid-shaped interventricular septum; HR, heart rate; SBP, systolic blood pressure; DBP, diastolic blood pressure; IVSBT, interventricular septal basal segment thickness; IVSMT, interventricular septal middle segment thickness; IVSBT/IVSMT, ratio of interventricular septal basal segment thickness to interventricular septal middle segment thickness; LVPWT, left ventricular posterior wall thickness; LVEF, left ventricular ejection fraction; LVOTD, left ventricular outflow tract diameter; LVOTV, left ventricular outflow tract flow velocity. 
Table 2. Comparison of longitudinal strain parameters of left ventricular segments between non-SIS group and SIS group (\%, $\bar{X} \pm$ s).

\begin{tabular}{|c|c|c|c|c|c|c|c|c|c|}
\hline \multirow{2}{*}{ Indicators } & \multicolumn{3}{|c|}{ Anterior septum } & \multicolumn{3}{|c|}{ Anterior wall } & \multicolumn{3}{|c|}{ Side wall } \\
\hline & Basal & Middle & Apical & Basal & Middle & Apical & Basal & Middle & Apical \\
\hline $\begin{array}{l}\text { non-SIS groups } \\
\quad(\mathrm{n}=30)\end{array}$ & $-13.63 \pm 2.45$ & $-21.5 \pm 6.99$ & $-20.5 \pm 8.63$ & $-18.37 \pm 5.48$ & $-17.13 \pm 6.33$ & $-15.38 \pm 8.96$ & $-20.63 \pm 1.92$ & $-20.13 \pm 5.22$ & $-18.37 \pm 5.8$ \\
\hline $\begin{array}{l}\text { SIS groups } \\
\quad(\mathrm{n}=30)\end{array}$ & $-8.25 \pm 2.34$ & $-21.7 \pm 4.59$ & $-23.92 \pm 5.55$ & $-18.08 \pm 5.53$ & $-14.17 \pm 5$ & $-13.17 \pm 7.94$ & $-19.67 \pm 3.26$ & $-17.33 \pm 7.58$ & $-21.08 \pm 5.16$ \\
\hline$P$ value & 0.000 & 0.924 & 0.293 & 0.909 & 0.259 & 0.57 & 0.304 & 0.378 & 0.288 \\
\hline \multirow{2}{*}{ Indicators } & \multicolumn{3}{|c|}{ Posterior wall } & \multicolumn{3}{|c|}{ Inferior wall } & \multicolumn{3}{|c|}{ Posterior interval } \\
\hline & Basal & Middle & Apical & Basal & Middle & Apical & Basal & Middle & Apical \\
\hline $\begin{array}{l}\text { non-SIS groups } \\
\quad(\mathrm{n}=30)\end{array}$ & $-18.38 \pm 3.9$ & $6-19.38 \pm 5.9$ & $9-21.13 \pm 2.9$ & $9-18.75 \pm 3.73$ & $-17.5 \pm 7.15$ & $-22.13 \pm 2.59$ & $-14.25 \pm 3.01$ & $-20.75 \pm 4.03$ & $-25 \pm 8.16$ \\
\hline $\begin{array}{l}\text { SIS groups } \\
\quad(\mathrm{n}=30)\end{array}$ & $-18.08 \pm 3.9$ & $6-18.42 \pm 5.4$ & $4-20.25 \pm 4.53$ & $3-13.67 \pm 6.58$ & $-21.42 \pm 4.2$ & $-22 \pm 6.71$ & $-9.08 \pm 3.12$ & $-20.58 \pm 5.99$ & $-26.17 \pm 4.65$ \\
\hline$P$ value & 0.874 & 0.712 & 0.636 & 0.064 & 0.139 & 0.961 & 0.006 & 0.946 & 0.688 \\
\hline
\end{tabular}

Note: Date are expressed as mean $\pm \mathrm{SD}$.

segment of the left ventricular anterior wall and the posterior wall of the left ventricle in the SIS group were significantly lower than those in the non-SIS group. The difference was statistically significant $(\mathrm{P}=0.000,0.006$, all $\mathrm{P}<0.05)$. However, there was no significant difference in other left ventricular segments and global LS between SIS group and non-SIS group $(P>0.05)$.

\section{Discussion}

In this study, the value of LS measured by 2D-STE was used to evaluate left ventricular regional and global systolic function of hypertensive patients with SIS, and to explore whether SIS affects left ventricular systolic function in hypertensive patients. Through the study, it was found that under the condition of normal LVEF, 2D-STE could early detect the abnormality of left ventricular regional myocardial longitudinal systolic function. Compared with hypertensive patients without SIS, SIS affects left ventricular regional systolic function in hypertensive patients.

SIS is a special morphological feature of the local thickening process to the left ventricular outflow tract at the base of the interventricular septum. Previous studies have shown that SIS patients are generally found in echocardiography, mostly in the elderly, especially in patients with hypertension [2] [3] [4]. So far, there is no consensus on the clinical significance of SIS [5]. For a long time, many scholars believe that SIS is a special anatomical structure formed in the process of cardiac aging, which is usually asymptomatic and of little clinical significance [1] [2] [3]. In 2009, the Framingham Heart Research Center [2] found 52 cases of SIS among 3562 participants in the community. During a 15-year 
follow-up, it was found that SIS was not associated with the risk of cardiovascular disease or death, indicating that SIS was not associated with poor prognosis. However, some case reports [8] [9] [10] [11] suggested that left ventricular hypercontraction is likely to occur under exercise load or when taking positive inotropic drugs, or when they are in postprandial or postural changes. In this case, SIS may cause left ventricular outflow tract obstruction, resulting in exertional dyspnea or syncope and other clinical manifestations, resulting in cardiovascular adverse consequences. Therefore, these scholars believe that SIS is pathological and has important clinical significance.

Previous studies have shown that there is a close relationship between SIS and hypertension [2] [3] [4]. With the aging of the population in China, more and more SIS have been found, especially in patients with hypertension. Due to the special anatomical structure of SIS, the hearts of these hypertensive patients with SIS may need to overcome more peripheral vascular resistance, further increase the cardiac afterload, cause high load work, and may lead to left ventricular overcontraction. In this case, the protruding base of the interventricular septum comes into contact with the active mitral valve lobe, which narrows the outflow tract, where the blood flowed converges to produce high-speed blood flow, which together promotes the increase in the pressure difference in the left ventricular outflow tract. It may cause left ventricular outflow tract obstruction and corresponding clinical manifestations, resulting in cardiovascular adverse consequences. Therefore, it is particularly important to evaluate the left ventricular systolic function and take effective measures to prevent the occurrence of cardiovascular adverse events in hypertensive patients with SIS as soon as possible.

As a non-invasive technique for quantitative evaluation of myocardial motion, 2D-STE has no angle dependence and is less affected by cardiac load, so it can accurately evaluate left ventricular myocardial motion function [6]. Among them, left ventricular myocardial LS has high sensitivity in evaluating local and global systolic function in cardiac subclinical stage [12]. Therefore, in this study, 2D-STE was used to measure left ventricular LS in hypertensive patients with SIS to evaluate left ventricular regional and global systolic function.

In this study, the results of conventional ultrasound measurement showed that the LVEF values of the were $(59.0 \pm 1.41) \%$ and $(59.14 \pm 2.59) \%$ in non-SIS groups and SIS groups respectively. There was no significant difference between the two groups $(\mathrm{P}>0.05)$. However, the LVEF values of the two groups were in the normal range $(50 \%-70 \%)$, indicating that the left ventricular systolic function was normal in both groups when evaluating left ventricular systolic function by conventional LVEF. Compared with non-SIS group, the values of IVSBT, IVSMT and IVSBT/IVSMT increased in SIS group, especially in IVSBT. However, the measured value of LVOTD decreased. The difference was statistically significant (all $\mathrm{P}<0.05$ ). The results showed that in the SIS group, except for the obvious thickening of the base of the interventricular septum, the thickness of the other ventricular wall was approximately normal, and the decrease of the inner diameter of the left ventricular outflow tract suggested that it might be re- 
lated to the occurrence of left ventricular outflow tract obstruction. The results of two-dimensional spot tracking showed that the LS of the basal segment of the anterior and posterior wall of the left ventricle in the SIS group was significantly lower than that in the non-SIS group. The difference was statistically significant (all $\mathrm{P}<0.05)$. However, there was no significant difference in other left ventricular segments and global LS between SIS group and non-SIS group $(P>0.05)$. That is, compared with the non-SIS group, the longitudinal deformation ability of the basal segment of the left ventricular anterior wall and the posterior wall of the left ventricle in the SIS group decreased, indicating that the left ventricular regional systolic function of the SIS group was decreased, which mainly occurred at the base of the interventricular septum. The reason may be related to the unique morphological characteristics of SIS.

\section{Summary}

In summary, compared with hypertensive patients without SIS, SIS affects the left ventricular regional systolic function in hypertensive patients, which mainly results in the decrease of myocardial longitudinal systolic function of the basal segment of interventricular septum. Therefore, SIS cannot be classified as a benign manifestation of cardiac aging. Under the condition of normal LVEF, the LS measured by 2D-STE can early evaluate the involvement of left ventricular regional systolic function. This is helpful for clinicians to evaluate hypertension patients with SIS as early as possible and take effective measures to control hypertension and its related risk factors, which has important clinical significance to prevent the occurrence of cardiovascular adverse events.

\section{Acknowledgements}

We thank the Department of Ultrasound of the first affiliated Hospital of Yangtze University for its experimental platform and technical support for this study.

\section{Conflicts of Interest}

The authors declare no conflicts of interest regarding the publication of this paper.

\section{References}

[1] Goor, D., Lillehei, C.W. and Edwards, J.E. (1969) The "Sigmoid Septum" Variation in the Contour of the Left Ventricular Outlet. American Journal of Roentgenology, 107, 366-376. https://doi.org/10.2214/ajr.107.2.366

[2] Diaz, T., Pencina, M.-J., Benjamin, E.-J., et al. (2009) Prevalence, Clinical Correlates, and Prognosis of Discrete Upper Septal Thickening on Echocardiography: The Framingham Heart Study. Echocardiography, 26, 247-253. https://doi.org/10.1111/j.1540-8175.2008.00806.x

[3] Canepa, M., Omar, M., Melissa, D., et al. (2014) Prevalence, Clinical Correlates, and Functional Impact of Subaortic Ventricular Septal Bulge (from the Baltimore Lon- 
gitudinal Study of Aging). The American Journal of Cardiology, 114, 796-802. https://doi.org/10.1016/j.amjcard.2014.05.068

[4] Gentille-Lorente, D. and Teresa, S.-U. (2016) Sigmoid Septum: A Variant of Ventricular Hypertrophy or Hypertrophic Myocardicity? Mexico Cardiology Files, 86, 110-122. https://doi.org/10.1016/j.acmx.2016.02.001

[5] Pearson, A.-C. (2017) The Evolution of Basal Septal Hypertrophy: From Benign and Age-Related Normal Variant to Potentially Obstructive and Symptomatic Cardiomyopathy. Echocardiography, 34, 1062-1072. https://doi.org/10.1111/echo.13588

[6] Mondillo, S., Galderisi, M., Mele, D., et al. (2011) Speckle-Tracking Echocardiography: A New Technique for Assessing Myocardial Function. Journal of Ultrasound in Medicine, 30, 71-83. https://doi.org/10.7863/jum.2011.30.1.71

[7] Hypertension Alliance (China), Cardiovascular Society of Chinese Medical Association (2019) Chinese Guidelines for the Prevention and Treatment of Hypertension Revised in 2018. Prevention and Treatment of Cardiovascular and Cerebrovascular Diseases, 1, 1-44.

[8] Sinclair, H.-C., Russhard, P., Critoph, C.-H., et al. (2019) Routine Orthostatic LVOT Gradient Assessment in Patients with Basal Septal Hypertrophy and LVOT Flow Acceleration at Rest: Please Stand up. Echo Research and Practice, 6, K1-K6. https://doi.org/10.1530/ERP-18-0072

[9] Hirata, Y., Hirotsugu, Y., Kenya, K., et al. (2018) Provocation of Clinically Significant Left Ventricular Outflow Tract Obstruction by Postural Change in Patients with Sigmoid Septum. Journal of Echocardiography, 16, 173-174. https://doi.org/10.1007/s12574-018-0372-x

[10] Umazume, T. (2013) Adverse Effects of Cilostazol on Left Ventricular Function in a Patient with a Sigmoid Shaped Interventricular Septum. International Journal of Cardiology, 165, 551-555. https://doi.org/10.1016/j.ijcard.2012.09.007

[11] Ozaki, K., Ikki, S., Koko, M., et al. (2008) Effect of Cibenzoline and Atenolol Administration on Dynamic Left Ventricular Obstruction due to Sigmoid-Shaped Septum. Circulation Journal, 72, 2087-2091.

[12] Imbalzano, E., Concetta, Z., Scipione, C., et al. (2011) Left Ventricular Function in Hypertension: New Insight by Speckle Tracking Echocardiography. Echocardiography, 28, 649-657. https://doi.org/10.1111/j.1540-8175.2011.01410.x 